\title{
A Jewelry Data QRCode Generation Service Based on Agent Technology
}

\author{
X. Y. Li \\ School of Mathematics and Computer Science \\ JiangHan University \\ Wuhan 430056, P.R. China \\ e-mail: 813809236@qq.com
}

\begin{abstract}
-for implementing the description of jewelry inspection data with two dimension code, based on agent technology, a QRCode generation service is proposed by encapsulating the two dimension code generation system running in the local network environment. The jewelry cert generation service can calls the QRCode generation service to generate jewelry cert QRCode. The practicability and validity of the QRCode generation service is verified through the application in the jewelry inspection business management system.
\end{abstract}

Keywords: Quick response code(QRCode); two dimension code;agent; jewelry inspection business management system

\section{INTRODUCTION}

For description the jewelry inspection information with two dimension code, in last years, a QRCode generation systems have been developed in author's laboratory, and it runs in the local domain network environment. [1] In recently, we have developed the jewelry inspection business management systems with visual studio net platform, and this system will run in the internet environment. In this condition, we must change the local QRCode generation system to adapt the internet environment. [2] In this paper, we encapsulate the local QRCode generation system as a common QRCode generation service based on agent technology. This service can accept soap message with $\mathrm{xml}$ format and generate the QRCode, and then the jewelry cert generate service can call the QRCode service to generate the QRCode which describes the jewelry inspection information and add the QRCode the jewelry inspection cert. [3]

The reminder of this paper is structured as in the following: section 2 gives an overview on QRCode; section 3 gives a brief introduction on the multi-agent encapsulation model. In section 4 discusses how to encapsulate the local QRCode generation system as web services based on multiagent technology, and finally, section 5 contains the conclusion.

\section{QRCODE OVERVIEW}

The QRCode is a trademark for the matrix barcode designed for automobile industry. Now, the system has become popular beyond the industry due to its fast readability and large storage capacity compared to traditional one dimension barcode. The QRCode consists of black models arranged in a square pattern on a different background. [1] The description data can be consisted of four kinds of data, or any kind of data with extension. In 1994, QRCode was invented by the Toyota subsidiary Denso Wave to track vehicles during the manufacturing process, and was originally designed to allow components to be scanned at high speed. [2] It has since become one of the most popular of two dimensional barcodes. [3] Unlike the traditional one dimensional barcode that was designed to be scanned by a narrow beam of light, the QRCode is detected as two dimensional digital image by an image sensor and is then digitally analyzed by a programmed processor. The processor locates the three distinctive squares at the corners of the image, and uses a smaller square near the fourth corner to normalize the image for size, orientation, and angle of viewing [4]. The small dots are then converted to binary numbers and validity checked with an error-correcting code. Originally designed for industrial uses, QRCode has become common in consumer advertising. Telephone users can install an app with a QRCode scanner that can read a displayed code and convert it to a URL directing the telephone browser to the website of a company, store or product associated with that code providing specific information. $[5,6]$

In the shopping industry, knowing what causes the consumers to be motivated when approaching products by the use of QRCode, advertisers and marketers can use the behavior of scanning to get consumers to buy causing it to be the best impact on ad and marketing design. [7, 8] As a result, the QRCode has become a focus of advertising strategy, since it provides quick and effortless access to the brand's website. [9] Beyond mere convenience to the consumer, the importance of this capability is that it increases the conversion rate, by coaxing qualified prospects further down the conversion funnel without any delay or effort, bringing the viewer to the advertiser's site immediately, where a longer and more targeted sales pitch may continue. Although initially used to track parts in vehicle manufacturing, QRCode is now used over a much wider range of applications, including commercial tracking, entertainment and transport ticketing, product/loyalty marketing, and instore product labeling. It can also be used in storing personal information for use by government. [10] An example of this is Philippines National Bureau of Investigation where NBI clearances now come with a QRCode. Many of these applications target mobile-phone users. Users may receive text, add a vCard contact to their device, open a Uniform Resource Identifier, or compose an e-mail or text message after scanning QRCode. [11] They can generate and print 
their own QRCode for others to scan and use by visiting one of several pay or free QRCode-generating sites or apps. QRCode storing addresses and Uniform Resource Locators may appear in magazines, on signs, on buses, on business cards, or on almost any object about which users might need information. Users with a camera phone equipped with the correct reader application can scan the image of the QRCode to display text, contact information, connect to a wireless network, or open a web page in the telephone's browser. This act of linking from physical world objects is termed hardlinking or object hyperlinking. QRCode may also be linked to a location to track where a code has been scanned. Either the application that scans the QRCode retrieves the geo information by using GPS and cell tower triangulation or the URL encoded in the QRCode itself is associated with a location. [12] In June 2011, the Royal Dutch Mint issued the world's first official coin with a QRCode to celebrate the centennial of its current building and premises. The coin was able to be scanned by a telephone and link to a special website with contents about the historical event and design of the coin. [13] This was the first time of a QRCode used on currency.

\section{SYSTEM ENCAPSULATION ARCHITECTURE BASED ON MULTI-AGENT TECHNOLOGY}

Based on multi-agent techniques, every function of jewelry inspection business management system can be encapsulated as a web service, which may have various interfaces for different functions. The multi-agent based encapsulation architecture is shown Fig.1.

External wrapper provides various interfaces to accept SOAP request, which can be managed by the client's power, and inner wrapper are developed for the jewelry inspection business management system in the institutions [14, 15]. The SOAP requests from online user are transmitted to external wrapper, and then retransmitted to inner wrapper. Then the inner wrapper decomposes the requests into a sequence of status values, according to which web service will change the state, and related web services are called to respond to the request. Finally the result should be sent back to external wrapper which in the same way sends it to the online user $[16,17]$.

\section{A. External Wrapper}

External wrapper accepts requests and cooperates with inner wrapper. Its main tasks are to analyze and decompose the requests, including creating service detailed lists, decomposing request to status switch, defining communications protocols and the format of input and output, registering and issuing web services in the UDDI [13].

External wrapper is closed to the actual operation platform, and access request through inner wrapper, external wrapper is used to establish and maintain the safe communication among different web services. When communication happens among different web services in the jewelry inspection business management system, the conversion between bytes and certain data type must be run to ensure platform-independent need. The communication information is described and defined uniformly in XML. [14]

\section{B. Inner Wrapper}

Inner wrapper needs to coordinate with external wrapper, and interact with local QRCode generation system. Therefore, they should be standardized and adaptable to the operation

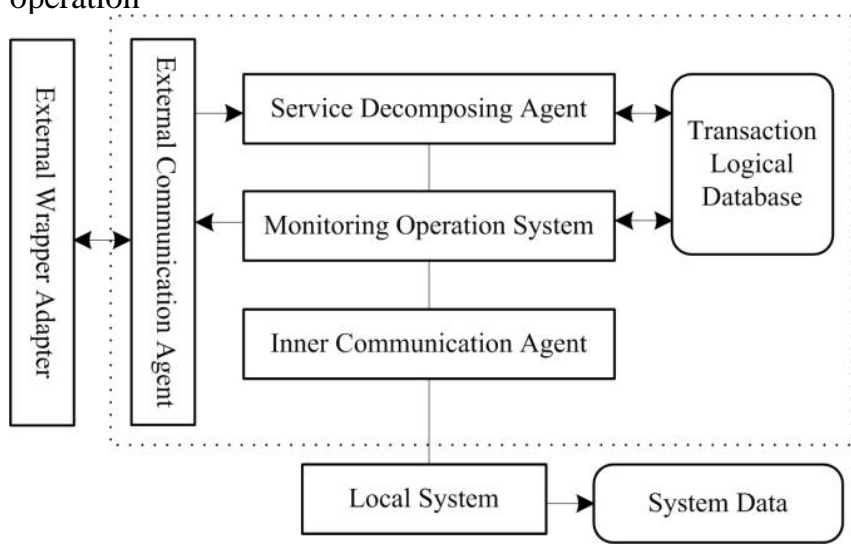

Figure 1. System encapsulation based on multi-agent technology

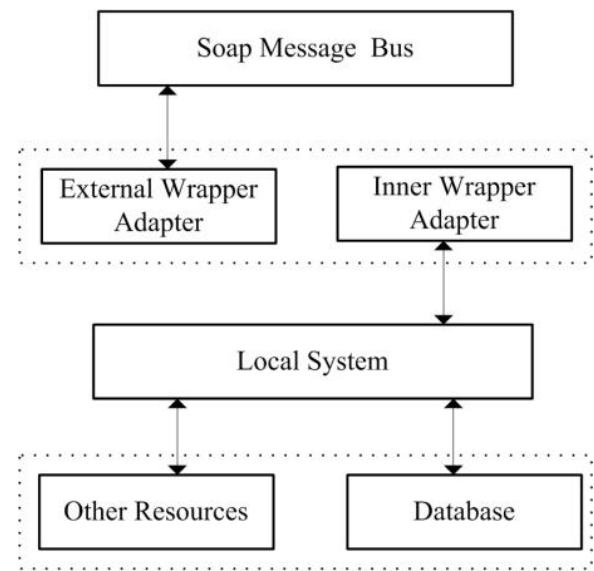

Figure 2. The architecture of inner wrapper

environments of local QRCode generation systems. The architecture of inner wrapper is shown in Fig. 2.

The net communication agent receives service request from external wrapper, and sends it to service decomposing agent, which decomposes the service request to the status switch set based on transaction logic library. The net communication agent is composed of external communication agent and inner communication agent. Then according to the status data, the monitoring operation agent manipulates related subsystems and sends the needed output to external communication agent.

\section{COMMON QRCODE GENERATION SERVICE}

The architecture of the common QRCode generation service is show in Fig.3. The Application server receives SOAP request from other services, and the jewelry inspection information is written to the real-time database, then the message monitor will find the data and send it to the local QRCode generation system, the main code of the local QRCode generation system is shown in Fig.5, the local 
QRCode generation system will generate the QRCode based on the received data and returns the QRCode image to the application server. Finally, the application server will send out the QRCode data with soap message.

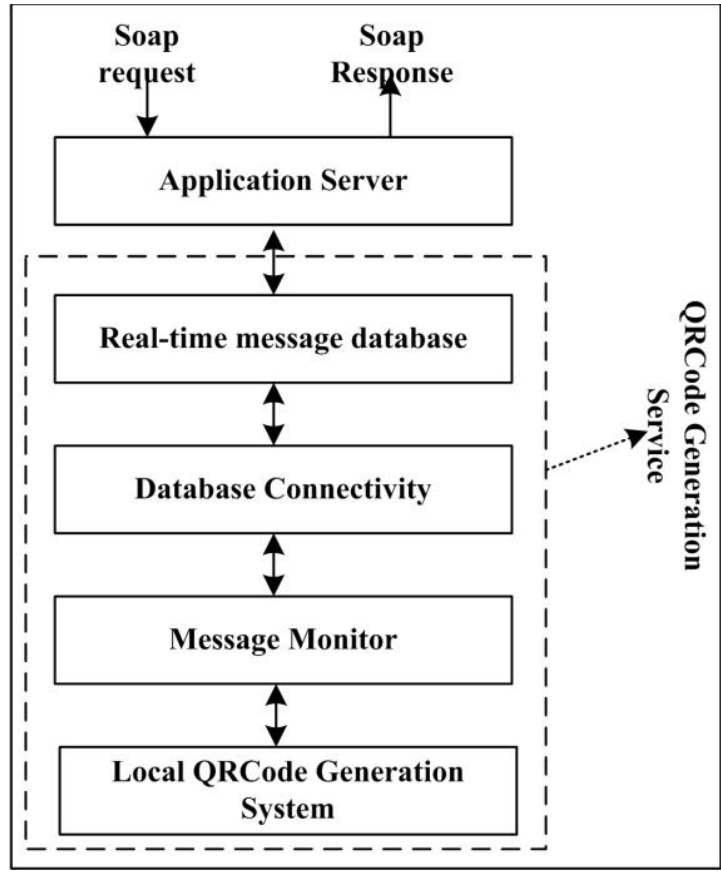

Figure 3. the common QRCode generation service

As show in Fig.4, according to different parameters, the local QRCode generation system can generate QRCode quickly. To the common QRCode generation service, all soap messages are described with $\mathrm{xml}$ format. As show in Fig.6, the QRCode generation soap request is described with $\mathrm{xml}$ format and is sent to the common QRCode generation service.

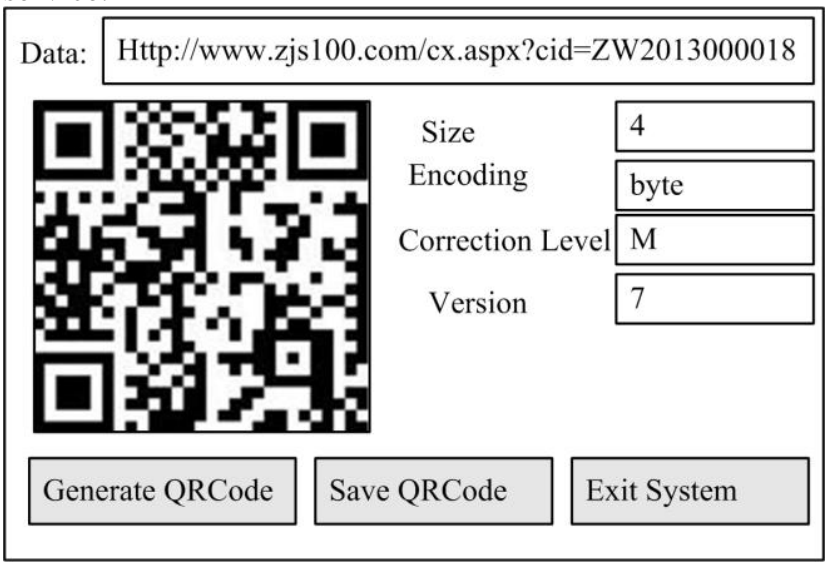

Figure 4. the generation of QRCode based on different parameters

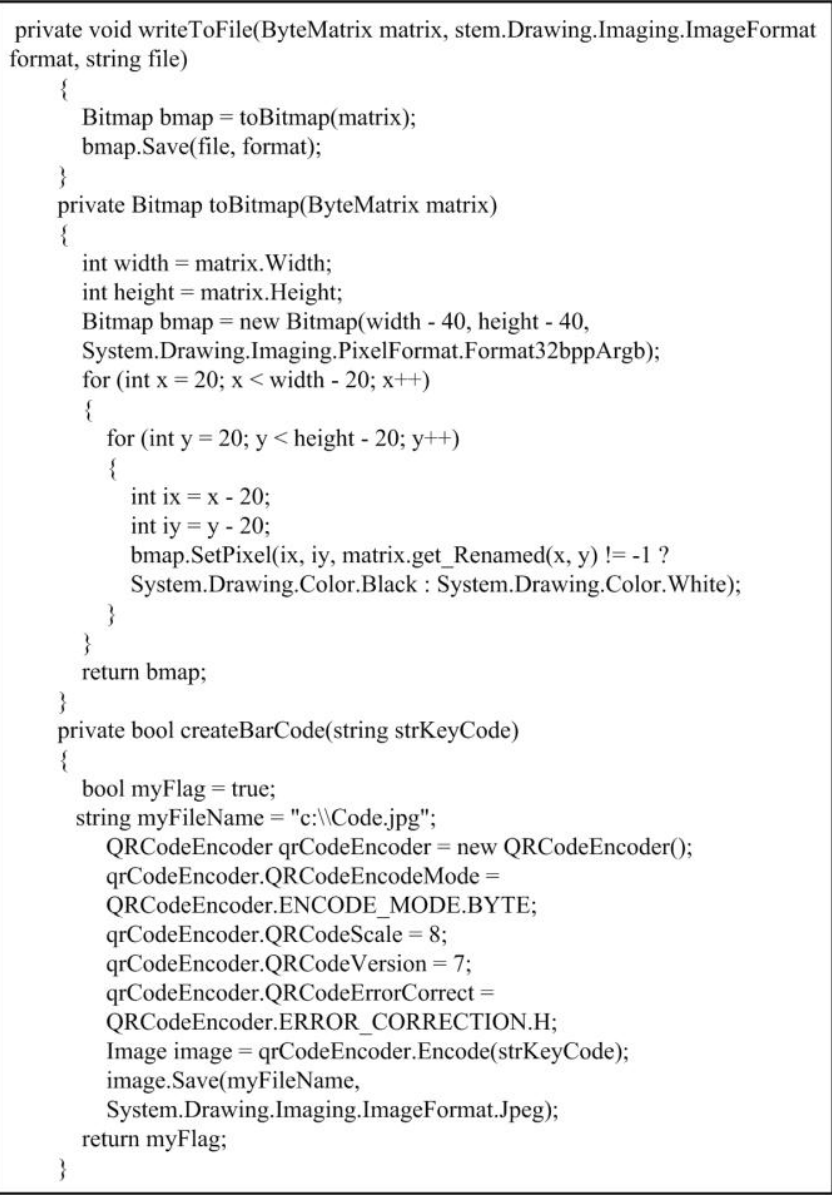

Figure 5. the main code of local QRCode generation system

POST /WebService1/QRCode_Service.asmx HTTP/1.1

Host: 114.80.101.47:909 Content-Type: text/xml; charset=utf-8

Content-Length: 524

$<$ ? $\mathrm{xml}$ version $=" 1.0$ " encoding $=$ "utf- 8 "? $>$

<soap:Envelope xmlns:xsi="http://www.w3.org/2001/XMLSchemainstance"

xmlns:xsd="http://www.w3.org/2001/XMLSchema"

xmlns:soap="http://schemas.xmlsoap.org/soap/envelope/">

$<$ soap:Body $>$

$<$ GQRcode $x$ mlns="http://tempuri.org/WS/QRCode_Service" $>$

$<$ Data $>$ www.ljzbw.cn/cx.aspx?sid=zw2013000455 $<$ /Data $>$

$<$ Encoding $>$ Byte $<$ /Encoding $>$

$<$ Correction Level $>\mathrm{M}</$ Correction Level $>$

$<$ Version $>7</$ Version $>$

$<$ Size $>4</$ Size $>$

$</$ GQRcode $>$

$<$ soap:Body $>$

$<$ soap:Envelope $>$

Figure 6. the SOAP message with XML format 


\section{CONCLUSION}

Based on agent technology, the QRCode generation service is implemented by encapsulating the QRCode generation system running in the local network environment. The service can accept and send SOAP message with $\mathrm{xml}$ format, and communicates with other services. The practicability and validity of the QRCode generation service is verified through the application in the jewelry inspection business management system.

\section{REFERENCES}

[1] T. Walker, "Making Best Use of QR codes: Gleaning Lessons from the Latest Data," Seybold Report: Analyzing Publishing Technologies,pp.2-7, 2011.

[2] J.K.Lee, "A study on authentication system using QR code for mobile cloud computing environment,"FutureTech 2011, pp. 500-507,2011.

[3] J.Gao, "Understanding 2D-BarCodes Technology and Applications in M-Commerce - Design and Implementation of A 2D Barcode Processing Solution," In: The Proceedings of COMPSAC 2007, vol. 2, pp.49-56,2007.

[4] M.Canadi, W.Hoepken, M.Fuchs, "Information and Communication Technologies in Tourism 2010: Application of QR Codes in Online Travel Distribution," In: Gretzel, U., Law, R., Fuchs, M. (eds.), vol. 4, pp.137-148. Springer, New York,2010.

[5] R.G. Ivancsits, "Mobile Couponing und Mobile TicketingInstrument des Customer Relationship Management in Mobile Marketing," Müller, Saarbrücken, pp.7-109,2006.

[6] W.L. Wang,"A Study of Two-dimensional Barcode Prescription System for Pharmacists Activities of NHI Contracted Pharmacy," Yakugaku Zasshi 128, pp.123-127,2008.

[7] Kuo, D., D.Wong, J.Gao, Chang, L.,"A 2D Barcode Validation System for Mobile Commerce," In: Chang, R.-S., Bellavista, P., Chao, H.-C., Lin, S.-F., Sloot, P.M.A. (eds.) GPC 2010. LNCS, vol. 6104, pp.150-161. Springer, Heidelberg ,2010.

[8] T. Kimura, M. Katoh,"Application System of the Universal Sign Code-Development of the Portable Sign Presenter," Lecture Notes in Computer Science Volume 5105, pp.678-681,2008.

[9] P. Grillo, S. Likavec, "Using Mobile Phone Cameras to Interact with Ontological Data," Lecture Notes in Computer Science Volume 6928, pp.568-576, 2012.

[10] J. Seo, " Imaged Based Codes Performance Comparison for Mobile Environments," Lecture Notes in Computer Science Volume 6761, pp.653-659, 2011.

[11] X. Liu, D. Doermann, "A Novel 2D Marker Design and Application for Object Tracking and Event Detection," Lecture Notes in Computer Science Volume 5358, pp.248-257, 2008.

[12] B.H. Kim, J.K. Lee, "A Study on Performance Evaluation of 7-Step QRCode Authentication Framework," Lecture Notes in Electrical Engineering Volume 164, pp. 449-457, 2012.

[13] Z. Liu, Q.L. Zeng, C.L. Wang, Q. Lu, "Application Research of QRCode Barcode in Validation of Express Delivery," Communications in Computer and Information Science Volume 143, pp.346-351, 2011.

[14] H.Y. Sun, "Multi-Agent dynamic fusion method based on utility evaluation for equipment intelligent diagnosis," Journal of Vibration Engineering, 22(4), pp.413-417, 2009.

[15] E. Serrano, "Infrastructure for forensic analysis of multi-Agent systems," Lecture Notes in Computer Science, pp.168-183, 2009.

[16] B. Fan, "Dynamic workflow management system supported by multiintelligent-Agent," Proceedings-2009 International Symposium on Information Engineering and Electronic Commerce, pp.192-196, 2009.
[17] F. Zhao, "A multi-Agent model for the order driven agile manufacturing systems and order form selection algorithm," 2009 IEEE International Conference on Information and Automation, pp.1625-1630, 2009. 